\title{
Le prix de la célébrité acquise par la transgression
}

\author{
Belfar BOUBAAYA NACIERA, Université Setif 2, Algérie
}

\section{Introduction}

Devenir célèbre peut être le résultat de circonstances variées. En effet, différentes conjonctures telles qu'un travail laborieux et soutenu, un héritage, une trouvaille précieuse, ou encore une invention lucrative peuvent rendre une personne célèbre. D'autres circonstances peuvent mener à la célébrité. Qu'en est-il de la célébrité dans le domaine de la littérature ? Est-il possible d'évoquer la célébrité acquise par des circonstances contraires à ce que les normes de vie et d'écriture prônent généralement? Qu'en est-il de la célébrité acquise par la transgression?

Transgresser avec l'implication de dépasser des limites, de rejeter un certain ordre ou encore de passer au-delà du respectable, du convenable ou même du divin est perçu comme une action négative. Un ordre établi et reconnu, respecté et pratiqué semblerait honorer ceux qui le célèbrent. Ainsi, rejeter ce même ordre et les règles qui y sont associées, expliquerait une mise à l'écart. Cependant, nous montrerons qu'une notoriété bénéfique peut percer malgré l'aspect de confrontation et de manquement à des valeurs normatives. C'est dans ce contexte que nous proposons de considérer la célébrité acquise par la transgression.

Charlotte Brontë et Assia Djebar sont deux écrivaines célèbres. Nous proposons de les présenter sous l'angle de la transgression positive, car elles illustrent la célébrité acquise par l'infraction aux normes requises à plusieurs niveaux. L'aspect transgressif de Djebar a été analysé par Silvia Nagy qui a joint la romancière à l'écrivaine Lemsine dans un article intitulé «Tradition and Transgression in the Novels of Assia Djebar and Aicha Lemsine ». Le terme de transgression est également rappelé par Jeanne-Marie Clerc dans son ouvrage Assia. Djebar : Ecrire, Transgresser, Résister. En ce qui concerne Brontë, le terme de transgression est associé à ses ouvrages par Claire Merias dans Ordre et désordre dans Jane Eyre de Charlotte Brontë. Merias rappelle que Jane Eyre «met en scène l'évolution de Jane et la romance avec son employeur, Rochester, liaison au départ décriée par la société victorienne », et définit l'héroïne comme «un personnage féminin qui défie sans cesse l'ordre établi » (1). L'auteure propose de considérer l'œuvre par rapport à « un ordre nouveau, [qui est] de détruire pour mieux rebâtir, même si l'on peut s'interroger sur ce qu'il advient à la fin de la révolte et de la transgression » (1). Pour cela, elle n'hésite pas à utiliser des titres et des sous titres tels que : «De l'ordre au désordre : la force transgressive du 
roman » (2) ou encore «L'amour ou la transgression » (4). L'analyse et le traitement de la transgression dans notre article diffèrent dans le sens où il s'agit de traiter de la montée vers la célébrité en mettant en exergue des aspects biographiques, sociaux et éducatifs, qui, dans la littérature victorienne de Brontë et la littérature algérienne de Djebar, sont en opposition avec la norme. Une approche biographique, historique et sociologique permet de relever des éléments de transgression que les écrivaines illustrent dans leurs romans.

Ces deux écrivaines rejettent certaines traditions nuisibles à l'écriture de par leur nature souvent liée à un esprit étroit. Des mentalités jugées préjudiciables peuvent être contrées. Devenir célèbre en commettant des transgressions conduit à considérer quels genres de dépassements ont pu permettre une notoriété dans le milieu littéraire. Chez les deux écrivaines, un dépassement est enregistré dès le moment où des actes ne s'inscrivent pas dans les normes requises par leurs sociétés respectives. Ce qui n'est pas habituel est considéré hors norme. La transgression peut être considérée comme déviance lorsque qu'il y a une opposition à l'ordre des règles à respecter (voir Becker). Les déviances constituent pour la sociologie un domaine particulier (voir Ogien). Mais la question qui se pose au niveau du conformisme vient du fait que ce qui n'est pas habituel, doit-il être qualifié de nuisible parce qu'il transgresse le requis? Il semblerait que trop pratiquer des actes devenus coutumiers, bien qu'ils puissent freiner une évolution et un bien-être, conduise à les accepter sans mettre en question leur dimension et leurs conséquences restrictives et négatives, surtout lorsque seulement une minorité d'individus est concernée. Or, et c'est le cas des deux écrivaines, transgresser des normes conduit à remettre en question un vécu comme faux, négatif, discriminatif, péjoratif et même destructeur. Ce qui est courant, c'est l'acceptation de normes sans questionnement sur leur légitimité. Brontë et Djebar ne se sont pas soumises à des normes négatives, restrictives et nocives. Par conséquent, la transgression qui peut être évoquée à leur égard, comprend des remises en question de pensée et de comportement envers les écrivaines.

Les romans de Brontë, traduits dans de nombreuses langues, ainsi que ceux de Djebar témoignent de leur renommée internationale. Des circonstances de rencontres littéraires ont permis à Brontë de côtoyer le célèbre écrivain William Thackeray qui dominait la scène littéraire victorienne. À Londres, en juin 1851, alors que Thackeray savait que Brontë était l'auteure de Jane Eyre, il lui demande, après avoir donné sa deuxième conférence dans la salle Willies, ce qu'elle pense de sa prestation, soucieux de la considération de l'écrivaine reconnue. La notoriété acquise était ainsi confirmée pour l'écrivaine novice. En ce qui concerne Djebar, de nombreux prix littéraires lui ont été 
attribués et témoignent de sa célébrité. Ses romans sont traduits en de nombreuses langues. De plus, elle eut l'honneur d'être la première femme algérienne nommée à l'Académie française (voir Bédarida). La célébrité des deux écrivaines est une réussite acquise après des efforts soutenus et contribue à un apport positif pour le monde de la littérature. Nous proposons d'observer quelques aspects de leur réussite sous l'angle du type de transgression positive à travers quelques citations tirées des œuvres des écrivaines. Notre corpus d'appui inclut quelques extraits des romans de Brontë, Jane Eyre (1847) et Villette (1853), ainsi que Femmes d'Alger dans leur appartement (1980), La femme sans sépulture (2002) et Nulle part dans La maison de mon père (2007) de Djebar. Dans la première partie, nous approchons les écrivaines d'un point de vue critique biographique. Quelques exemples de transgression positive provenant de la vie de Brontë et de Djebar sont considérés notamment dans le milieu éducatif. Dans la deuxième partie, nous proposons quelques illustrations des traitements de la transgression dans les romans. L'aspect historique de l'évolution de la société permettra de voir des transformations positives concernant l'expression des sentiments tels qu'illustrés par les personnages et le thème du mariage.

Cet article ne prétend nullement traiter tous les aspects de transgression très nombreux dans les œuvres des deux écrivaines. Le but réside plutôt dans l'illustration de l'acquisition de la célébrité acquise par un rejet de conventions. De par son aspect universel, la littérature révèle des entraves identiques qui peuvent se dresser autour d'une écrivaine dans deux sociétés différentes, à des époques différentes, avec des religions et des langues différentes. Une similarité réside dans le fait que nous traitons de deux sociétés patriarcales.

\section{Aspects de transgression dans la vie des écrivaines.}

De nos jours, les transgressions d'alors semblent totalement déraisonnables. Ainsi, afin de mieux comprendre où l'acte de transgression peut être qualifié de tel, un bref rappel de quelques normes requises dans la société des deux écrivaines est nécessaire. En Angleterre, dès le moment où Brontë se met à écrire en vue de publier, elle est consciente des obstacles auxquels elle aura à faire face. L'Angleterre victorienne est une Angleterre où l'homme domine tous les milieux. Fille de pasteur, Brontë grandit silencieusement sous la domination d'un monde au service du masculin. Elle bénéficie d'une éducation à la maison, car toute fille doit avoir une formation de femme au foyer en vue de devenir une épouse modèle. Le perfectionnement de l'épouse exemplaire à qui aucun reproche ne doit être fait quant à la conduite d'un foyer sans problème, était un objectif primordial. Brontë fréquente l'école des enfants de pasteur, une institution peu onéreuse. La jeune femme reste très 
insatisfaite de son statut social qui réduit les possibilités d'atteindre de plus grandes connaissances. Elle s'instruit avec succès en lisant tout ce qui lui passe sous la main, notamment tout ce que son père rapporte à la maison. Journaux, magazines, romans lui permettent de comprendre ce qui se passe dans le monde politique, religieux et aussi littéraire. Avide de romans en anglais, elle est au courant des auteurs qui dominent la scène littéraire et de leur style. Soucieuse de maitriser la langue française, elle reçoit des romans en français d'une amie, Nussey, avec qui elle correspond en français, et dévore les ouvrages. Pour gagner un peu d'argent, Brontë instruit les enfants de gens aisés. Maltraitée et mise à l'écart en tant que gouvernante, elle rejette cette profession ingrate et pense plutôt à ouvrir une école privée pour filles qui lui permettrait d'accéder à une meilleure situation. Etre financièrement indépendante lui permettrait de sortir du milieu dans lequel elle vit et de l'étroitesse dont elle est consciente.

Mais atteindre un tel but implique qu'il faut sortir des normes requises habituelles. Pour bénéficier d'études plus poussées que celles offertes à sa condition, il faut aller à l'étranger où Brontë désire perfectionner sa maitrise de ce français qui est un atout pour une école privée. Elle demande à sa tante maternelle de lui payer des études en Belgique. Cette tante a toujours été aux côtés de ses nièces depuis la mort de leur mère, très jeune, afin de leur assurer une bonne éducation. Cependant, les jeunes filles victoriennes ne suivent pas habituellement des études à l'étranger seules. Ceci est encore moins courant pour une fille d'un statut social comme celui des filles Brontë. Chez les gens aisés, un professeur vient d'ordinaire à la maison pour éduquer les filles. Les enfants de familles aisées s'adonnent aux langues, à la broderie, à la peinture et à la musique. Ce ne sont pas des filles de la condition de Brontë et ses sœurs qui vont à l'étranger pour s'instruire. Brontë est hors norme, car elle et ses sœurs ne peuvent pas se permettre un tuteur à la maison. L'ambition de la jeune femme est plus forte que le respect des conventions. À Bruxelles, elle désire perfectionner son français et aborder d'autres domaines.

La Belgique expose la jeune fille à bien des situations hors règle. Pour le premier voyage d'Angleterre à Bruxelles en 1842, Brontë est accompagnée par son père Patrick Brontë et par une amie aisée Mary Taylor qui elle-même est avec son frère, car une jeune fille respectée ne voyage pas seule dans la société victorienne. Mais quand Brontë doit retourner en Angleterre subitement à l'annonce de la mort de sa tante, elle rentre seule avec sa sœur et entreprend un voyage singulier. Deux jeunes filles sans la compagnie d'un homme qui voyagent si loin, était un fait très rare. De plus, après l'enterrement de sa tante, c'est sans Emily qu'elle repart à Bruxelles, car celle-ci ne supporte plus d'être loin de la maison. Brontë 
se retrouve donc seule dans le pensionnat dirigé par Monsieur et Madame Héger. Il lui est proposé d'enseigner en échange des cours qu'elle suit dans cette école. Elle donne même des cours d'anglais à son professeur de français, qui n'est autre que Monsieur Héger. À la fin de ses études, elle rentre à nouveau seule à la maison paternelle.

Anxieuse d'améliorer sa situation financière, Brontë arrive à convaincre ses sœurs, qui s'adonnent à l'écriture comme elle, de publier leurs manuscrits. Brontë est consciente du fait qu'elle ne peut publier que sous un pseudonyme masculin. Elle réunit ses travaux et ceux de ses sœurs et les convainc d'envoyer leurs productions sous les pseudonymes masculins de Currer Bell, Acton Bell et Ellis Bell ; les jeunes écrivaines voulaient protéger leur identité par rapport à une société qui considérait que la place des femmes était au foyer et non pas dans le monde du marché littéraire (Burkhart 48). Les écrivaines ne seraient pas acceptées et pourraient même être rejetées pour toujours si leurs écrits paraissaient sous leur vrai nom de femme. Ainsi, l'écriture des jeunes femmes fait connaitre leurs ouvrages et suscite la curiosité des cercles littéraires qui s'interrogent sur l'identité de ces bons écrivains inconnus jusque-là. Le succès est immédiat; sans cette transgression, les sœurs Brontë ne seraient pas devenues célèbres.

La transgression s'inscrit ici dans un rejet de l'impossibilité de s'exprimer en tant que femme. Les règles habituelles présentent des écrivains hommes comme Thackeray, Robert Southey, Charles Dickens et bien d'autres dans une société patriarcale où ils ont tous le droit d'écrire et de publier. Face aux auteurs femmes, l'homme semblait se sentir dérangé, perturbé et même menacé dans un domaine où il prônait la domination et la supériorité. En contexte, faute de généralisation d'écriture féminine, cet acte est considéré comme une tentative de sortir des normes. Il n'était pas de l'éthique victorienne d'avoir des écrivaines au même titre que les écrivains. Une mise au défi de normes et d'ordre sécurisants est lancée à la société par des écrivaines, dont Brontë et ses sœurs. Admiratrice de Southey, Brontë lui écrit en mars 1837 pour lui demander des conseils. Il lui répond qu'écrire n'est pas du domaine des femmes (voir Bloom 6). Southey demande ironiquement s'il n'y a pas de chaussettes à raccommoder avec un frère ou même un mari dont Brontë devrait s'occuper. Elle répond qu'elle est consciente de ce rôle à tenir mais qu'en parallèle elle peut également écrire (Deirdre vii.). Brontë rejette l'idée du privilège d'écrire que l'homme s'est approprié. Elle déclare qu'elle a observé tous les devoirs qu'une femme doit remplir (Gilbert et Gubar 63).

Une autre forme de transgression s'inscrit dans la correspondance que la jeune femme entretient avec son professeur de français de Bruxelles, Monsieur Héger, homme marié, à qui elle s'attache. Bien des doutes ont été soulevés quant à la raison des lettres 
échangées et sur les limites de l'amitié qui sembleraient avoir été dépassées. Brontë exprime dans sa correspondance un profond attachement à son ex-professeur. Elle le prie de croire qu'en lui écrivant, il fait une bonne action, qu'elle espère recevoir de ses nouvelles qui la tranquilliseront et apaiseront sa tristesse, et qu'il est un doux plaisir pour elle de voir son écriture (Gilbert et Gubar 605). Très vite, Madame Héger réagit et Monsieur Héger cesse cette correspondance. Brontë en souffre profondément. Elle redouble d'efforts dans l'écriture de ses romans (voir Ernest sur la publication de Jane Eyre, 270).

L'Algérie où grandit Djebar est une Algérie sous la colonisation française d'abord, puis une Algérie indépendante. Djebar de son vrai nom Fatima Zohra Imalayène, va à l'école à Cherchell, son village natal, puis à Mouzaïa, loin de chez elle. Elle est différente des autres petites filles du village où elle a grandi. Dans l'organisation scolaire de l'enfance de Djebar, une petite fille ne va pas à l'école publique, car ceci contredit la pratique courante. Une fillette algérienne ne peut aller qu'à une école coranique. Pour Djebar, c'est grâce à la transgression opérée par son père qu'elle a accès à une éducation en langue française. Benjamin Prémel estime que «grâce au père, elle a été 'libérée' de cet enfermement promis aux autres filles arabes » (98). Le père de Djebar, Tahar Imalayène, est instituteur de français, formé à l'école normale de Bouzaréah. Alors qu'il supervise une classe de cinquante-trois garçons dans sa ville, il fait entrer sa fillette dans le monde masculin. Il est persuadé du fait qu'acquérir des connaissances et une langue dominante est un point positif. Il désire que sa fille soit instruite au même titre qu'un garçon. Étant la seule petite fille de son école, Djebar ressent une impression de transgression confirmée lorsque son père l'appelle pour qu'elle se joigne aux cinquante-trois élèves, tous garçons, pour la pose de la photo scolaire de fin d'année (voir Calle-Gruber, 255. Djebar est placée au deuxième rang et son père se tient sur le côté). La transgression des normes opérée par le biais du père est très particulière car celuici est un homme instruit, attentif à agir de façon sage et réfléchie. Son statut d'instituteur lui permet de s'imposer et d'imposer ses actes. Son éducation incite au respect chez ses concitoyens qui ne peuvent pas critiquer ou se moquer de sa décision. La notion d'acte tabou est rejetée, les préjugés sont tus et la critique sociale est freinée.

Le succès scolaire conduit Djebar à partir seule en France où elle poursuit ses études. Elle est la première femme algérienne à l'Ecole normale supérieure de Sèvres. Elle se consacre à l'écriture et produit son premier roman, La soif, en 1957. Le pseudonyme que l'écrivaine choisit, a pour but de ne pas heurter sa famille. Très vite, écrire et décrire les mœurs bourgeoises et les intrigues amoureuses de la société algérienne désignent la jeune romancière de femme singulière et osée. Par la suite, Djebar se rend compte que son écriture 
devient trop autobiographique alors qu'une femme arabe ne parle pas d'elle. Katarina Melic souligne que «Dans la société musulmane algérienne, écrire pour une femme, c'est intervenir contre quelque chose, contre le monopole des hommes. Un écrivain-femme commet une transgression qui est le fait d'écrire, de prendre la parole. Prendre la parole est un geste audacieux ». De plus, comme l'indique Latifa Sari Mohamed, «L'écriture chez la femme est souvent cachée, occultée. Elle est considérée comme une transgression. Il s'agit non seulement de transgresser l'interdit de toute écriture, mais encore de le transgresser par rapport à l'homme, par rapport à la société »(87-96). Quand Djebar écrit son deuxième roman, Les alouettes nä̈ves, elle inscrit le même type de transgression car «tout aussi inhabituelles pour ne pas dire scandaleuses sont les militantes [de ces deux derniers romans], dans la mesure où sortant de l'ombre - dans tous les sens du mot - où elles se sont toujours tenues, elles s'affirment presque en égales de l'homme. . . Assia Djebar semble bien être la seule, parmi les romanciers algériens qui ont décrit la guerre d'indépendance, à y avoir montré des femmes dans un rôle autre que secondaire » (Sari 87-96)

Rejeter des restrictions dans le cas de Djebar tout comme dans celui de Brontë leur a permis de devenir célèbres.

\section{Formes de transgression dans les romans d'Assia Djebar et de Charlotte Brontë}

Les instances de transgression proposées ici sont illustratives de différentes thématiques dans le rejet de normes requises. Le clivage social et ses codes, les sentiments amoureux difficiles à exprimer dans la société victorienne et la société algérienne, le voyage en solitaire pour une femme, les effets vestimentaires à observer et l'impact de l'éducation témoignent d'une conduite transgressive pour les deux romancières dans un milieu patriarcal. Brontë expose le lecteur victorien à de nouvelles normes. Une échappée de conventions est présente et permet de secouer les traditions. Les comportements exigés des femmes sont très respectueux et révèlent des contraintes oppressantes. Le désir féminin est honteux. Ce dont on ne doit pas parler en public fait surface dans les romans de l'écrivaine. Dans Jane Eyre, lorsque de vrais sentiments se développent entre un employeur hautain, sévère et ironique et une gouvernante que la norme ne permettrait jamais d'épouser, l'écrivaine insiste sur le fait que Jane Eyre est une femme avant d'être une gouvernante. La véhémence des émotions provoquées par la douleur des sentiments blessés donne libre cours à une plaidoirie touchante exprimée par l'héroïne. Elle est magnifiquement présentée mais elle constitue une transgression chez une femme qui exprime des désirs féminins ouvertement et appartient à une classe sociale inférieure à celle de l'aimé. Selon Nancy Amstrong, le désir ne peut pas 
être supprimé et il ne le sera pas ; comme le roman victorien ne reconnait pas le désir féminin comme un aspect humain, il ne peut pas être placé dans la catégorie de la culture de la classe moyenne (110).

Dans le roman, Jane Eyre ne contrôle plus ses sanglots, qui disent sa profonde peine, et ne maîtrise plus ses mots. Elle a jusque-là toujours réprimé ce qui ne convient pas à une bonne conduite aux normes victoriennes. C'est à travers le personnage de Madame Fairfax, intendante du manoir de Monsieur Rochester à Thornfield, que Brontë verbalise l'interdit que s'impose une femme posée et conservatrice. Madame Fairfax est étonnée de voir la respectueuse Mademoiselle Eyre exposer ses sentiments et rappelle à la jeune femme que les gentlemans du statut de son employeur n'ont pas coutume d'épouser leur gouvernante (249). L'écrivaine exprime des sentiments profonds, car elle-même a souffert du clivage social et ne s'est pas permis d'épouser Georges Smith Taylor, son éditeur plus riche qu'elle, de peur d'entraver les coutumes du mariage. Dans le roman, la gouvernante finit par épouser son employeur et transgresse ainsi les convenances de la société victorienne. Pour atténuer l'interdit, Brontë crée un héritage qui permet à Jane Eyre de s'élever au rang social de Monsieur Rochester. Le couple heureux et solidaire illustre un côté positif de la transgression.

Elizabeth Gaskell, écrivaine victorienne qui a rédigé la biographie de Brontë, ne permettra pas à ses filles de lire Jane Eyre à cause de l'immoralité de la trame amoureuse où les mœurs sont transgressées. Rebecca Frazer mentionne cet aspect en utilisant le terme «naughty $(\mathrm{xv})$ ou «vilain » pour décrire le roman interdit. Le traitement des sentiments et de l'amour chez Brontë est hors du commun. La lecture en groupe si habituelle au coin de la cheminée par les Victoriens ne pouvait permettre la lecture de Jane Eyre. A cette époque, la lecture était une activité familiale au sein des foyers de la classe moyenne; les membres d'une même famille se relayaient pour une lecture partagée à haute voix ; le texte à lire devait donc être conforme à l'écoute de chacun et ne pas comporter de points non inacceptables ou inadaptés à chacun des auditeurs (Flint 24). L'héroïne du roman, trop passionnée, représente un mauvais exemple et un modèle dangereux pour les jeunes filles. En d'autres mots, sa passion, en particulier la colère de Jane Eyre face aux contraintes de la société, horrifiait les Victoriens, dont la morale était stricte et rigoureuse (Gilbert et Gubar 338). La censure de tels aspects romanesques était de coutume.

En même temps, l'écrivaine est consciente des limites religieuses à ne pas dépasser. Elle ne permet pas la transgression des valeurs du mariage. Dans Jane Eyre, bien que Monsieur Rochester ait été dupé dans un premier mariage malheureux avec une femme folle, 
il se voit refuser la proposition immorale et illégale qu'il fait à Jane Eyre. Il lui offre de devenir sa maitresse, c'est-à-dire de transgresser les liens licites du mariage en allant habiter en France où personne ne les connait et où ils pourraient vivre heureux. L'héroïne ne se permet pas de transgresser les liens divins du mariage et Jane Eyre prend la fuite.

Ce type de transgression religieuse est également fortement présent chez Djebar. Dans Femmes d'Alger dans leur appartement, Toumi fait une fugue amoureuse avec le consentement de sa mère qui s'assure auparavant que sa fille se mariera religieusement et légalement avant de consommer sa nuit de noce. La religion est évoquée pour que Toumi soit protégée et acceptée dans la société algérienne. La dimension des actes amoureux à ne pas rendre publics est abordée par Djebar à travers le personnage de Fatima dans Nulle part dans la maison de mon père qui commet une transgression en fréquentant un garçon en cachette. À travers le côtoiement de filles françaises et d'algériennes émancipées, la narratrice découvre des actes amoureux. De plus, la proximité d'un établissement pour garçons l'expose à de jeunes intellectuels. La tentation d'avoir un amoureux conduit la jeune femme à fréquenter en cachette. Selon le code des convenances, une fille algérienne ne se mêle pas à l'autre sexe. Fatima craint d'être découverte par son père lorsqu'elle prétexte des sorties à l'université pour en fait rencontrer son ami. Consciente de la transgression des pratiques conservatrices de sa famille, elle s'éloigne au maximum de la maison dans des lieux que son père ne fréquente pas. Si son père venait à découvrir cette fréquentation, elle s'auto-punirait : « La phrase tragique ou mélodramatique met en branle son tic-tac effréné en toi. Si mon père le sait, je me tue ! (412). En même temps, la narratrice reconnaît : «je n'ai accepté que des baisers qui ne m'ont même pas émue ni bouleversée, mais la transgression et le risque pris,...» (431). Ces faits illustrent le degré problématique de la transgression. Dans l'épilogue du roman, la narratrice précise encore : "'Si mon père le sait, je me tue !' une telle alarme, hyperbole de la transgression alors qu'elle ne se sent coupable que d'avoir accepté des baisers » (444).

Ces actes de transgression sont connus pour être contraires aux lois de la société, du père et du village où la narratrice se rend pour les vacances chaque été et qui lui rappelle d'où elle est originaire. Ce même village expose la narratrice à une autre forme de transgression, car d'autres dépassements sont entrepris en secret par les jeunes filles qui rêvent d'un amoureux et qui transgressent les interdits en écrivant des lettres secrètes aux adresses qu'elles trouvent dans les journaux et magazines. Elles contreviennent aux règles sociales. La transgression que se permet Djebar, est l'évocation directe de la relation des sexes. Ce traitement, dans l'écriture de Djebar, diffère de l'écriture de bien des romanciers algériens, 
car elle va au-delà de ce que l'on rencontre habituellement dans la littérature algérienne où une gêne se crée quant aux sentiments amoureux affichés ouvertement. En contexte algérien, pour Djebar, une transgression de normes est un constat réel.

Une autre forme de transgression dans les sentiments amoureux qui ne peuvent pas s'afficher ouvertement, trouve une échappatoire dans le thème de la fugue amoureuse mentionnée chez les deux écrivaines. Fuguer semble être une solution dans une relation rejetée et impossible dans les deux sociétés où les écrivaines ont évolué. Dans Jane Eyre, Georgiana Reeds, personnage secondaire, se rend à Londres avec sa mère et sa sœur. Très belle, Georgiania est admirée par le jeune Lord Edwin Vere, qui tombe amoureux d'elle; mais les proches du jeune homme ne sont pas d'accord sur le choix d'une jeune fille de classe sociale inférieure à la leur. Devant leur amour rejeté, les jeunes gens décident de fuguer et Georgiana, surveillée par sa sœur, est dénoncée à sa mère et vite rattrapée (Brontë 86). Fuguer est également une solution dans Villette, où la jeune et belle Ginevra Fanshawe est anxieuse d'épouser celui qui lui permettra d'accéder à une classe sociale supérieure à celle qu'elle occupe. Elle fugue avec le jeune colonel Dehamel, dont le statut social est attirant. Pour corriger sa transgression, son oncle, plus aisé que son père, lui octroie une rente ; ceci permet au mariage d'être approuvé.

Dans Femmes d'Alger dans leur appartement, la jeune Toumi commet le même genre de transgression, car elle fugue pour se marier contre le gré de ses frères, ses tuteurs à la suite de la mort du père. Les frères refusent le prétendant et Toumi est révoltée. Sur les conseils de sa mère, la jeune fille fait approuver son mariage par les liens religieux. Consciente de l'importance de la religion, Djebar présente ce mariage comme une réussite, car l'un des frères de Toumi lui demandera d'élever son fils à la suite du décès de sa femme. Toumi mène une vie conjugale apaisée et sa famille lui rendra visite après un reniement requis par les normes de la société patriarcale.

Les deux écrivaines semblent exprimer les mêmes notions de reconnaissance de transgression avec des valeurs positives. Djebar traite de sujets considérés comme étant hors norme et parfois même tabous. Elle expose les droits du coutumier qu'il faut respecter malgré le mal engendré et y oppose le côté positif de la transgression. Ce genre d'écriture se rapproche de ce qu'affirme Brontë dans Jane Eyre, dans lequel la notion des bons usages est remise en question. Le conformisme qui s'affiche en société n'est pas la morale dans de nombreuses situations. Les conventions contraignantes semblent devoir être révisées et même parfois rejetées. 
La transgression chez Brontë et Djebar peut aussi être analysée dans le cas de ce qui est permis du point de vue vestimentaire ou encore des aspirations à une éducation considérée comme hors norme pour une fille victorienne ou une fille algérienne. Les deux auteures utilisent toutes deux le français avec le point commun qui est la facilité de transmettre des états sentimentaux. La narratrice dans Villette révèle en français ce qui ne se dit pas en anglais. Quant à Djebar, elle précise à travers Fatima, narratrice de Nulle part dans la maison de mon père, que le français peut transmettre des sentiments amoureux qui ne se disent pas en arabe. La langue française dans le contexte victorien tout comme dans le contexte algérien, où l'arabe est le moyen de communication, devient un outil de transgression au niveau linguistique.

Apparait ainsi une déconstruction de la tradition en tant que force oppressive chez Djebar et Brontë. Les deux écrivaines indiquent des aspects de deux sociétés patriarcales à travers leurs œuvres et décrivent une rébellion contre ce qui est préjudiciable à certains personnages. Les héros et les héroïnes des romans reflètent une personnalité du changement de comportement vers une ouverture d'esprit pour trouver le bien-être. La transgression entreprise à leur niveau vise une amélioration. Les écrivaines trouvent dans la fiction une possibilité de s'exprimer à propos de ce qu'elles ne peuvent pas dévoiler dans la réalité. La transgression de normes dans le comportement et le langage est ainsi rendue possible dans la fiction. Comme le dit Freud, la fiction permet à l'artiste de traiter de ce qu'il ne peut pas traiter dans la réalité. (Wellek et Warren 82). Opérer des transgressions dans des romans pourrait-il faire prendre conscience de la réalité à laquelle aspirent les écrivaines ? Le laps de temps qui s'écoule entre le temps de l'écriture et le monde actuel, produit déjà une réponse car bien des situations exposées dans les romans sont désormais dépassées. La transgression considérée comme telle au moment de l'écriture pourrait ne plus être regardée comme transgression au moment de la lecture. La littérature reste un terrain d'investigation de changement de degré de transgression dans bien des aspects. La transgression positive telle qu'illustrée dans la célébrité des écrivaines, reste une quête d'amélioration de nombreuses situations diminutives. Le chercheur et le critique littéraire trouvent à leur disposition des œuvres qui restent à exploiter afin de refléter l'évolution des normes dans bien des domaines.

\section{Bibliographie}

Amstrong, Nancy. «Gender and the Victorian Novel». The Cambridge Companion to the Victorian Novel. Dir. David Deirdre. Cambridge : Cambridge UP, 2007.

Becker, Howard S. «Whose Side Are We On? » Social Problems 14.3 (1967) : 239-247. 
Bédarida, Catherine. «L'Académie française ouvre ses portes à Assia Djebar ». Writing as Resistance : Assia Djebar's Vaste est la prison. Le Monde. 18 juin 2005.

Bloom, Harold. The Brontës. New York : Chelsea, 2008

Brontë, Charlotte. Jane Eyre. Londres: Smith and Elder, 1847.

---. Villette. Londres : Smith and Elder, 1853.

Burkhart, Charles. Charlotte Brontë : A Psychological Study of her Novels. Londres, 1973.

Calle-Gruber Mireille. Assia Djebar ou la résistance de l'écriture, regard d'un écrivain d'Algérie. N.p. : Maisonneuve et Larose, 2001.

Clerc, Jeanne-Marie. Assia. Djebar : Ecrire, transgresser, résister. Paris : Harmattan, 1997.

Deirdre, David. Intellectual Women and Victorian Patriarchy. Londres : The Macmillan P, 1987.

Djebar, Assia. La soif. Paris : Julliard ,1957.

---. Nulle part dans la maison de mon père. Paris : Fayard, 2007.

---. Femmes d'Alger dans leur appartement. Paris : Femmes, 1980.

---. La femme sans sépulture. Paris : Albin Michel, 2002.

Ernest, Raymond. In the Steps of the Brontës. Londres : Rich and Cowan, 1948.

Flint, Kate. The Cambridge Companion to the Victorian Novel. Cambridge: Cambridge UP, 2007.

Frazer, Rebecca. Shirley and the Professor. N.p. : Everyman's Library, 2008.

Gilbert, Sandra et Susan Gubar. The Madwoman in the Attic, the Woman Writer and the Nineteenth Century Literary Imagination. New Haven et Londres : Yale UP, 1979.

Melic, Katarina. «L'exil et/ou la recherche d'une langue littéraire, Assia Djebar ou le blanc de l'écriture ». Mots Pluriels. 17 avril 2001.

http://www.arts.uwa.edu.au/MotsPluriels/MP1701km.html

Merias, Claire. Ordre et désordre dans Jane Eyre de Charlotte Brontë. Cercles : Occasional Papers, 2010.

Nagy, Silvia. Tradition and Transgression in the Novels of Assia Djebar and Aicha Lemsine. Research in African Literatures 33.3 (Automne 2002).

Ogien, Albert. La Sociologie de la déviance. Paris : Colin, 1985.

Prémel, Benjamin. «Lire, écrire, ... s'écrire ! L'école comme lieu d'apprentissage(s) dans les œuvres d'Assia Djebar et de Leila Sebbar ». Mémoire de Master. Paris : U Paris III Sorbonne nouvelle, 2011.

Sari Mohamed, Latifa. «La parole occultée ou le voile du silence dans Oran, Langue morte d'Assia Djebar ». Synergie Algérie 3 (2008). 
Wellek, René et Warren Austin. Theory of Literature. A Seminal Study of the Nature and Function of Literature in all its Contexts. Harmondsworth : Penguin, 1963. 